\title{
Assessment of Quality of Life of Family Caregivers of Patients Suffering from Stroke
}

\author{
Sabrina $^{1 *}$, Dr. Nasar Sayeed Khan ${ }^{2}$, Kanwal Qaiser ${ }^{3}$, Dr. Mansoor Ghani ${ }^{4}$ \\ ${ }^{1,3}$ RN, RM, Post RN BSN, MSN, Senior Nursing Lecturer, Institute of Nursing, Wah Medical College, Wah Cantt Pakistan \\ ${ }^{2}$ Associate Professor, Director Faculty Development, Department of Psychiatry, Queens University, Kingston on Canada \\ ${ }^{4}$ MBBS, MPHIL, MHPE, Associate Professor, Biochemistry, University of Health Sciences, Lahore Pakistan
}

DOI: $10.36348 /$ sjnhc.2020.v03i01.002

| Received: 08.01.2020 | Accepted: 15.01.2020 | Published: 22.01.2020

*Corresponding author: Sabrina

\section{Abstract}

Objectives: To investigate the Quality of Life (QOL) of Family care givers and characteristics of QOL associated with caregivers who are involved in the care of individuals with Cerebro Vascular accidents patients. Research Methodology: Descriptive cross-sectional study was conducted with a sample size of 50 caregivers of stroke patients taken from four different hospitals of Lahore. Non-probability convenient sampling technique was used. Data was collected by questionnaire of assessment of Quality of Life of family caregivers translated into Urdu version. Findings and Discussion: A Significant high ratio of female caregivers was found, out of 50 caregivers only 20 (40.0\%) were male. According to data, the average score of Social relationship domain score $63.7 \pm 18.4$ was followed by Psychological 58.8 \pm 15.1 and Physical health domain score $57.4 \pm 17.6$ as compared to the Environment domain score. The environment domain score $53.5 \pm 15.4$ was lowest as compared to all four domains' score. Conclusion: Findings of this study suggested that environmental domain is a leading factor for poor Quality of Life. It is needed to improve the environment and surroundings of patient care premises to improve quality of life of caregivers in hospital. Hospital policy, resources, set up and certain interventions were required to improve the caregivers' quality of life.

Keywords: Stroke; Caregivers; Burden; Quality of life.

Copyright @ 2020: This is an open-access article distributed under the terms of the Creative Commons Attribution license which permits unrestricted use, distribution, and reproduction in any medium for non-commercial use (NonCommercial, or CC-BY-NC) provided the original author and source are credited.

\section{INTRODUCTION}

Stroke is the most prevalent cause of morbidity and mortality all over the world. Many of stroke survivors are living with certain disabilities and need help in performing activities of daily living. There are few communities based epidemiological studies which were conducted in Pakistan on stroke. Stroke is second leading cause of death worldwide and is one of the leading causes of disability in developed and under developed countries [1].

It is one of the major causes of death and deprivation from health status in Pakistan. It is an important cause of morbidity and mortality. According to a fact sheet of WHO [2], it is the second leading cause of death worldwide. It is also observed that about five million deaths and more than 15 million non-fatal events occur each year, among 50 million survivors many of them are suffering from recurrent attacks of stroke [3].
Pakistan is sixth most populous country in the world with an estimated population of approximately 167 million by July 2008. According to World Health Organization estimation, 5.5 million people died of stroke in 2002, and roughly $20 \%$ of those deaths occurred in South Asia. Estimated annually about 350,000 cases of stroke reported in Pakistan the Express Tribune, October $29^{\text {th }}, 2015$. The tyranny of the disease in South Asian countries (India, Pakistan, Bangladesh, and Sri Lanka) has inclined and is expected to rise [4].

The involvement of family caregivers in providing care to stroke patient is very widespread in Asia, because it involves the cultural errands, morals, religious values and spiritual satisfaction among family members. According to Linda L Pierce in 2001[5], individuals and families always strive for well-being and they assume caring is a part of spirituality and wellbeing and is a precursor of love, affection, values and beliefs. 
By considering the cultural values, beliefs, roles and responsibilities of caregivers this change in role cannot be derelict. A family caregiver is requisite for scrupulous to meet the needs of patient, by considering this role of caregivers, shift in responsibilities occur and consequently this brings a drastic change in their quality of life [5].

An additional study recommended that training of caregivers not only perks up patient outcomes but also help them to provide cost effective medical care. The economic burden they are facing during long term stay in hospital can also be minimized by giving training and educating the caregivers regarding specific techniques of handling the client. Total health and social care cost reduce as income is a major factor which is directly associated with the quality of life[6].

\section{LITERATURE REVIEW}

According to World Health Organization, "Quality of Life is individual's perception of their position in life in the context of the culture and value systems in which they live and in relation to their goals, expectations, standards and concerns. It is a broad ranging concept affected in a complex way by the person's physical health, psychological state, level of independence, social relationships, personal beliefs and their relationship to salient features of their environment" [2].

In Asia and other countries informal care givers or family caregivers have an indispensable role in patient care. According to a Cape Town study Informal caregivers are those who endow with amateur care for the elderly or for people require physical, emotional, social and financial assistance with dependability in the home and spend much time and energy for long periods of time[7]

Caregivers affirm with this routine and their function declines according to the circumstances and role distorted. A decline in the neurological functioning of a patient is related to the consequences and burden of caregiver during their stay in hospital. Along with stroke patient, caregiver quality of life is also affected in many ways. Their present state changes not because of disease but due to change in role and responsibility[4].

Transformation of role and responsibility brings the change in quality of life. If the caregiver have poor health and lower income, and being a spouse having negative impact on quality of life. During the long stay in hospital influence the, physical, mental, social and psychosocial state turn down. These all are considered as important predictor for decreasing quality of life of care giver[8].

The contributory factors which are reported by caregivers are family matters, social life, financial constraints and lack of availability of resources during their stay in hospital. When the caregivers do not receive support and formal training, this may result in stress and negative health consequences which can additionally contribute to poor patient rehabilitation outcomes. Social and economic constraints are experienced by client caregivers [3].

Now a day, the role of a caregiver is considerably increasing. Similarly, the caregivers are also experience emotional agony. Hence, the patient as well as caregiver can face calamitous consequence due to this burden. In a previous research Researchers develop a model to study the quality of life of the caregiver in the relationship to their environmental factors, existing situations, and characteristics of the caregiver [9].

The responsibility of family is substantial in recuperation of patient particularly those family caregivers who is unswervingly contribute in patient care and endeavor to espouse the hospital environment changes, where they are not facilitated and may not get resources to fulfill their own needs. When a patient is anguish from stroke, the caregivers are allied with encumber or strain, which impinge on the quality of life of not only the caregiver but the stroke survivor as well. Here, a well notion out plan is required to diminish the saddle of the caregiver [10].

Quality of life of client and caregivers' satisfaction are directly interrelated, if there is high quality of life there will be higher satisfaction regarding care and provision of treatment. This satisfaction can be provided by need assessment of both, when the caregiver seems to be in the role of care provider his needs must be addressed. Provision of support and dealing them with kindness reduces the chance of dissatisfaction among patient and their caregivers. Provision of information and communicating regarding patient care is necessary in treating patient disease [11].

\section{METHODOLOGY}

Qualitative descriptive study is a design that is recognized for its plasticity. The study population included participants from three major hospitals Jinnah Hospital Lahore, General Hospital, Services Institute of Medical Sciences including general ward and private rooms which takes 6 month of study duration including the Total participants were 50 informal family caregivers.

\section{Inclusion Criteria for caregivers}

- Caregivers of stroke patients who are confined to bed.

- $\quad$ Age 20-45 years

- Both gender (Male /female)

- All Caregiver spend almost 2 weeks with patient 9-10 hours /day 


\section{Exclusion Criteria}

- All other family members who were not involved in patient care. (Visitors)

- Age more than 45 years

\section{DATA COLLECTION}

Data was collected from all participants in the study by considering the inclusion and exclusion criteria. Informed consent given, permission granted and clear explanation given to each participant. Confidentiality indemnified to protect the ethical rights of the participants. All the participants in the study willingly consented to take part in the study. A questionnaire WHOQOL-BREF was used to assess Quality of life of caregivers who were providing care to patients having stroke and stays in hospital approximately for two weeks. Khan MN et al. [1] conducted a study to proof that the WHOQOL-Bref questionnaire to identify quality of life is valid tool and was translated in Urdu especially for Asian population

\section{DATA ANALYSIS}

Data was analyzed by SPSS Software version IBM-20 by using and presented in the form of tables, percentage and frequencies for qualitative variables and linear regression.

\author{
Variables \\ Demographic variables \\ - Age \\ - Gender \\ - Socioeconomic status \\ - Education
}

\section{Indicators for Quality of Life \\ - Physical Domain \\ - Psychological Domain \\ - Social Domain \\ - Environmental Domain \\ - Poor QOL < 50\% \\ - Good QOL 75\% \\ - High QOL> 75\%}

\section{RESULTS}

The section of results comprises of descriptive statistics and demographic profile of caregivers and stroke patient, rest of the results indicate quality of life of caregivers, Its all associated domains like physical, psychological, social relationship and environmental respectively. In this study, a total of 50 family caregivers $(n=50)$ who were providing care to stroke patients were selected. The mean age of the patients was $59.9 \pm 14.8$. Majority of the patients were females and only $15(30.0 \%)$ were males.

Table-1: Caregivers perception about quality of life

\begin{tabular}{|c|c|c|c|c|c|c|}
\hline Q. No. & \multirow[t]{2}{*}{$\begin{array}{l}\text { How would you rate } \\
\text { your quality of life? }\end{array}$} & $\begin{array}{c}\text { Very poor } \\
\text { n }(\%)\end{array}$ & $\begin{array}{l}\text { Poor } \\
\text { n(\%) }\end{array}$ & $\begin{array}{l}\text { Neither poor nor } \\
\operatorname{good} \mathbf{n}(\%)\end{array}$ & $\begin{array}{l}\text { Good } \\
\text { n(\%) }\end{array}$ & $\begin{array}{l}\text { Very good } \\
\text { n }(\%)\end{array}$ \\
\hline Q.1 & & - & $10(20 \%)$ & $20(40 \%)$ & $18(36 \%)$ & $2(4 \%)$ \\
\hline \multirow[t]{2}{*}{ Q.2 } & \multirow[t]{2}{*}{$\begin{array}{c}\text { How satisfied are you } \\
\text { with your health? }\end{array}$} & $\begin{array}{c}\text { Very } \\
\text { dissatisfied } \\
n(\%)\end{array}$ & $\begin{array}{c}\text { Dissatisfied } \\
\mathbf{n}(\%)\end{array}$ & $\begin{array}{c}\text { Neither satisfied } \\
\text { nor dissatisfied } \\
n(\%)\end{array}$ & $\begin{array}{c}\text { Satisfied } \\
\text { n(\%) }\end{array}$ & $\begin{array}{l}\text { Very } \\
\text { satisfied } \\
n(\%)\end{array}$ \\
\hline & & - & $10(20 \%)$ & $16(32 \%)$ & $20(40 \%)$ & $4(8 \%)$ \\
\hline
\end{tabular}

The first question was "How would you rate your quality of life?" The response to this question showed that $2(4 \%)$ caregivers agreed on the option very good, $18(36 \%)$ caregivers agreed on option good, $20(40 \%)$ the caregivers agreed on the option neither good nor poor but only $10(20 \%)$ caregivers were agreed on option poor.

Though the life satisfaction is difficult to explain in words but assessing quality of life is a measure to assess satisfaction in life. There is a significant relationship of life satisfaction with all four domains of quality of life [12]. The second question was "How satisfied are you with your health?" The response to this question showed that $4(8 \%)$ caregivers indicated option very satisfied, $20(40 \%)$ caregivers indicated the option satisfied, 16 (32\%) caregivers indicated the option neither satisfied nor dissatisfied, but only $10(20 \%)$ caregivers indicated option dissatisfied and no one indicated the option very dissatisfied.

Table-2: Mean score and distribution of Quality of Life Score

\begin{tabular}{|c|c|c|c|}
\hline Quality of Life & Mean \pm S.D & Minimum & Maximum \\
\hline WHOQOL-BREF Questionnaire Score & $84.7 \pm 13.9$ & 50 & 120 \\
\hline $\begin{array}{l}\text { WHOQOL-BREF Questionnaire } \\
\text { Score in Percentage }\end{array}$ & $65.1 \pm 10.7$ & 38.5 & 92.3 \\
\hline \multicolumn{4}{|l|}{ WHOQOL-BREF Domains: } \\
\hline Physical health & $57.4 \pm 17.6$ & 31 & 94 \\
\hline Psychological & $58.8 \pm 15.1$ & 19 & 88 \\
\hline Social Relationship & $63.7 \pm 18.4$ & 19 & 100 \\
\hline Environment & $53.5 \pm 15.4$ & 13 & 100 \\
\hline
\end{tabular}


It is a 26-items self-report instrument designed to measure multidimensional quality of life (QOL) with maximum score of 130 . Higher scores for the scales and subscales indicate better quality of life. The overall mean score of quality of life measured by WHOQOLBREF questionnaire was $84.7 \pm 13.9$ with range from 50 to 120 . The overall mean score percentage of quality of life was $65.1 \pm 10.7$ with range from 38.5 to 92.3 . According to data, the average score of Social relationship domain was followed by Psychological and Physical health domain score as compared to the Environment domain score. The environment domain score was lowest as compared to all four domains' score.

Table-4.8

\begin{tabular}{|c|l|l|}
\hline WHOQOL-BREF Score Level: & Frequency & Percentage \\
\hline$<50 \%$ (Poor) & 5 & 10.0 \\
\hline $50-70 \%$ (Moderate) & 31 & 62.0 \\
\hline$>70 \%$ (Good) & 14 & 28.0 \\
\hline
\end{tabular}

The score of WHOQOL-BREF questionnaire is also categorized as: less than $50 \%$ score indicates poor quality of life, $50 \%$ to $70 \%$ indicates moderate quality of life and score $>70 \%$ indicates good quality of life. There were only $14(28.0 \%)$ caregivers who had score $>70 \%$, majority of caregivers $31(62.0 \%)$ had score between 50 to $70 \%$ whereas, $5(10 \%)$ had score < $50 \%$.

\section{DISCUSSION}

Quality of life is a subjective perception of each individual towards one's wellbeing. It is a complex phenomenon which is assimilated into four domains includes Physical, social, Psychological and environmental. These domains carrying different standpoint, including physical domain is related with subject of activities of daily living, dependence on medicinal substances and medical aids, energy and fatigue, mobility, Pain and discomfort sleep and rest. Psychological domain answers the questions regarding Bodily image and appearance, Negative and positive feelings, self-esteem spirituality/ religion/ personal beliefs and thinking, learning, memory and concentration. In social domain social relationships includes social support sexual activity.

In this research the first two questions were related to individual perception and satisfaction about quality of life. Average response was found regarding these questions, very few caregivers indicated the option dissatisfied and no one indicated the option very dissatisfied. Majority of the target population perception about quality of life is that, only $2(4 \%)$ caregivers agreed on option very good, 18 (36\%) caregivers agreed on the option good, $20(40 \%)$ of the caregivers were neutral.

According to study, the average score of Social relationship domain was high as followed by Psychological and Physical health domain score as compared to the Environment domain score. Total $63 \%$ of total population gets social support which helps them to increase satisfaction in relationships. The most effected was environmental as its score was lowest $53 \%$ as compared to all four domains' score. The considerable part of environment is financial resources freedom, physical safety and security health and social care: accessibility and quality Home environment opportunities for acquiring new information and skills participation in and opportunities for recreation/leisure activities physical environment (pollution/noise/traffic/climate) transport all are considerable factors which contribute in low or poor quality of life.

The findings of the study show average impact on the quality of life of caregivers. According to responses, the average score of Social relationship domain was followed by Psychological and Physical health domain score as compared to the Environment domain score. The environment domain score was lowest as compared to all four domains' score. According to a study on the subject of quality of life on a scale of 0 to 100 , the values for the domains that had values above 50 are considered to be a good perception of quality of life, which shows good scores Miranzi et al.

The caregiver who is deprived of the situation may lose the social connections and experience isolation. Presence of social support helps to mitigate with the situations, daily progress and change in the perception of being alone. Literature shows clear evidence of direct relationship of social support with caregiver. If there is amplified burden on caregivers, then this will decrease in social relationship. Those caregivers who are involved in the care of elderly people may experience emotional distress. It is observed in a study that the higher social support leads to higher satisfaction among caregivers $[13,14]$.

Fourth domain which is environment includes financial resources, freedom, physical safety and security in their surroundings. This domain also associated with health and social care: accessibility and quality, their home environment, opportunities for recreation / leisure activities, transportation and environment pollution / noise / traffic / climate.

In this study socio demographical data shows there are more female care givers involved in care and their age, education, level of income and daily life 
activity more compromised. A study conducted by [7, 15] which reflect the socio demographic profile directly intermingle with care giver burden. Care giver age, gender, education, income all affect them. Low income leads to stress and majority of care giver were older. Majority of care givers in this study with secondary education, but literature proves that education contributes to understand patient condition and cope up with situation.

At the stage where patient's all activities like eating, bathing, hygiene, movement become the task for caregivers, the caregivers vary with their tendency to handle those difficulties. Similarly, gender wise capacity to handle patient and providing care is a difficult task. As the results in table 1.1show that the most of the patients admitted with stroke were female. It has been shown in table 4.3 results, that the higher ratio of caregivers in hospital was females.

In current study social domain score was high. According to questionnaire social domain consist of family, friend's social support and relationship. Sense of safety and satisfaction seems associated with social support. Majority of the people answered that they get support from family and friends and their social relationship providing them satisfaction. It was difficult to ask the question related to their sexual life because of culture barrier, gender role and people were reluctant to answer this question.

The most questionable domain is physical because change in the situation may limit their physical boundaries and movement in routine. Caregivers sleep, rest, dependency, comfort, energy to handle patient and activities of daily living are the most affected areas. It is ultimate fact that, while staying in hospital physical needs are compromised. Physical empowerment and age both are considerable factor. In this study, the majorities of the caregivers were female, and considered as less physically stronger as compared to men. Most of the females were performing role of a caregiver. Here a female physical strength is questionable for adapting the situation.

The Psychological domain associated with the positive and negative feelings were found connected with hopelessness, helplessness joy, happiness. Personal perception of situation is usually defining positive feelings. Negative feelings include the sadness, guilt, lack of pleasure in life. Thinking memory, perception spirituality, religious belief, learning memory is also part of this domain.

This domain is related to the economic status, self-safety and security, convenience to resources, time for leisure activities, transportation facility, domestic environment and opportunity to obtain new information and skills. In this research, this domain gets significantly gets the lowest score as compared to the other domains.

Though, the discourse analysis in this study predicted poor or low quality of life among caregivers of stroke patient. Certain interventions and planning should be imparted for those situations and try to improve quality of life of client and caregiver too.

\section{CONCLUSION}

The results presented in this study showed the relationship of quality of life along with all four domains and identified the feature which are directly associated with all four domains of quality of life. In regards to different domain which influence and contribute to poor quality of life, Results of this study helps in understanding different characteristic associated with all four domains of quality of life, which are directly related and effecting quality of life.

On the other hand, hospital policy, facilities, structure, resources and protocols for nursing management must have an impact on reducing burden and improving quality of life. These multi-dimensional domains need to be addressed for specific interventions target population. In this regard it is the prime responsibility of nursing and other health care professionals to assist the caregivers in handling their difficult situations.

It is suggested that, social support, training and education programs must be initiated to deal with the situation. Limitation of resources directly influences the psychological domain. However, there is limitation of intervention which is directly associated with factors. In this regard more, research is required in this field to acknowledge a caregiver's issues and intervention and guidance plan needs to be addressed for improvement of quality of life of caregivers.

\section{SUGGESTIONS}

- The health professionals who are directly dealing with patient and informal caregivers must pay attention towards caregiver's needs and experience being an informal caregiver; this could be integrated with the health care domain and multidisciplinary team.

- Different strategies and training session must be arranged within hospital premises to teach caregiver to relieve physical, social, spiritual worries.

- Health care provider must be well trained to lower the burden of caregiver in hospital setting. They must teach caregiver easy and simple techniques to handle a stroke patient.

- Different rehabilitation teaching sessions should be arranged, complete information regarding patient condition and outcomes should be shared with caregiver. 
- There is an obvious need to confer the problems faced by caregivers and address their issues and integrate the caregivers for better outcome of client and reduce the risk of poor quality of life.

- There is dire need to develop policies and set of organization to make environment less irritating for caregiver and patient both so they can stay in hospital.

- Provision of visiting room according to the patient number in hospital.

- Provision of resources, medication, food for patient, hygiene and specially caregiver stay in hospital and their needs must be addressed.

\section{REFERENCES}

1. Khan, M.N. (2003). Translation and validation of quality of life scale, the brief version. Journal of Physicians Surg Pak. Feb 13

2. WHOQOL Bref. (2018). Measuring Quality of Life, WHO/Msa /Mnh / Psh /97.4

3. Barbara Campos de Oliveira. (2011). Caregivers of people with stroke needs, feeling and guidelines provided, 24(1) Sao paulo

4. Bhojo. A. Kheealani. (2008). Stroke in Pakistan, Journal of Pakistan medical association stroke, 58(7).

5. Linda I Pierce. (2001). Caring and expression of spirituality by urban care givers of people with stroke in African American Families.

6. Anita Patel, Martin Knapp. (2004).Training caregivers of stroke patients: Economic evaluation BMJ volume 328 .
7. Yakubu, A. Yakubu. (2018). Care givers attribute and socio demographic determinants of care giving burden in selected low income communities in cape town south Africa, Journal of compassionate health care 2018

8. Yeon- Gyu Jeong. (2015). Modifying role of care giver burden on predictor of quality of care givers of hospitalized chronic stroke patients.

9. White, C.L.I. (2004). Quality of life restrains toward a model of quality of life fofr family care givers of stroke survivor. April, 13(3): 625-38

10. Witness, M. (2010). Impact of care giver education on stroke survivor and their care givers

11. Jane, M, Cramm. (2012). Satisfaction with care as a quality of life predictor for stroke patients and their care givers.

12. Yildrim, Y.I. (2013). Relationship between life satisfaction and quality of life in Turkish nursing school students. Epub Jan 2

13. Fernanda, A. (2011). Influence of social support on the quality of life of family care givers while caring their patient

14. Hussain, N.A. (2014). Predictor of life satisfaction among family care givers of of hospitalized first ever stroke patients ASEAN Journal of Psychiatry, 15

15. Tatiana Ferreira da Costa. (2015). Quality of life of care givers for patients of cerebrovascular Accident: Association of socio demographic characteristic and burden. 